\title{
Arborescences
}

Revue d'études françaises

\section{Nous/on : De la réalité linguistique à la salle de classe}

\section{Alain Thomas}

Numéro 5, juillet 2015

Acquisition du français L2

URI : https://id.erudit.org/iderudit/1032665ar

DOI : https://doi.org/10.7202/1032665ar

Aller au sommaire du numéro

Éditeur(s)

Département d'études françaises, Université de Toronto

ISSN

1925-5357 (numérique)

Découvrir la revue

Citer cet article

Thomas, A. (2015). Nous/on : De la réalité linguistique à la salle de classe.

Arborescences, (5), 126-138. https://doi.org/10.7202/1032665ar

\section{Résumé de l'article}

Le remplacement du pronom nous par on, fréquent en français moderne, est encore peu reconnu dans l'enseignement du FL2 au niveau universitaire canadien. Pour mieux comprendre ce retard pédagogique sur la réalité linguistique et contribuer à le réduire, nous ferons d'abord ici une synthèse des études consacrées au sujet, après un rappel historique de l'évolution des deux pronoms. Une analyse de la présentation de la première personne du pluriel dans les manuels scolaires permettra ensuite de réexaminer nos pratiques pédagogiques, non seulement pour la paire nous/on, mais aussi pour d'autres aspects du français « ordinaire ", qui sont mieux acceptés dans la pratique quotidienne des locuteurs que dans le français soigné des grammairiens prescriptifs. d'utilisation que vous pouvez consulter en ligne.

https://apropos.erudit.org/fr/usagers/politique-dutilisation/ 


\section{SOMMAIRE}

Jeffrey Steele et Mihaela Pirvulescu. Université de Toronto Introduction

Jean-Marc Dewaele. University of London

07 Gender Errors in French Interlanguage: The Effect of Initial Consonant versus Initial Vowel of the Head Noun

Emily Felker, Annie Tremblay et Peter Golato. Université d'Utrecht, Université du Kansas et Université d'État du Texas Traitement de l'accord dans la parole continue chez les apprenants anglophones tardifs du français

Caitlin Gaffney. Université de Toronto L'acquisition L2 des compléments infinitifs français par des apprenants anglophones

Martin Howard. University College Cork

97 At the Interface between Sociolinguistic and Grammatical Development: The Expression of Futurity in L2 French - A Preliminary Study

Alain Thomas. Université de Guelph Nous/on : de la réalité linguistique à la salle de classe 


\title{
Nous/on: De la réalité linguistique à la salle de classe
}

\author{
Alain Thomas. Université de Guelph
}

\section{Résumé}

Le remplacement du pronom nous par on, fréquent en français moderne, est encore peu reconnu dans l'enseignement du FL2 au niveau universitaire canadien. Pour mieux comprendre ce retard pédagogique sur la réalité linguistique et contribuer à le réduire, nous ferons d'abord ici une synthèse des études consacrées au sujet, après un rappel historique de l'évolution des deux pronoms. Une analyse de la présentation de la première personne du pluriel dans les manuels scolaires permettra ensuite de réexaminer nos pratiques pédagogiques, non seulement pour la paire nous/on, mais aussi pour d'autres aspects du français "ordinaire», qui sont mieux acceptés dans la pratique quotidienne des locuteurs que dans le français soigné des grammairiens prescriptifs.

Il n'y a pas si longtemps, on considérait encore officiellement que l'emploi de on pour nous appartenait à la langue non standard de niveau populaire. Maurice Grevisse n'affirmait-il pas, dans ce classique de la grammaire française qu'est Le bon usage, que " certaines substitutions de on à nous ne sont parfois que des vulgarités de langage "(Grevisse 1969 : paragr. 587) ? Mais les tendances populaires d'hier sont souvent devenues le français ordinaire d'aujourd'hui et force est de constater qu'à l'heure actuelle, l'emploi de on en référence à plusieurs personnes bien déterminées est devenu monnaie courante, même dans les milieux cultivés, à tel point que l'usage de nous est de plus en plus réservé à la langue écrite ou aux registres les plus soignés de l'oral. On peut se demander ce qui a pu motiver ce glissement sémantique qui, a priori, ne fait que compliquer les choses : pronom singulier mais à sens pluriel, problème d'orthographe (Ma mère et moi, on est arrivé[s ?] hier soir), niveau de langue, et ainsi de suite.

Pour tenter de répondre à cette question, nous commencerons par un rappel historique permettant de mieux comprendre l'évolution du phénomène. Nous passerons également en revue les études qui documentent l'usage actuel de ce on nouvelle formule, tant en France que dans la francophonie canadienne, pour mieux mesurer l'écart qui sépare les observations de grammairiens comme Grevisse de la réalité linguistique contemporaine. Nous examinerons ensuite comment les manuels utilisés actuellement à l'université présentent la première personne du pluriel (dorénavant 1PL) aux étudiants. Cette analyse permettra enfin de se demander dans quelle mesure il faut enseigner la variante on et, d'une façon plus 
générale, de réexaminer l'enseignement du français " ordinaire " au niveau universitaire.

\section{Définitions}

L'opposition nous/on examinée ici est limitée à la position clitique sujet et au sens restreint de "je + autres connus " (Boutet $1986: 1$ ), c'est-àdire les contextes où le locuteur a le choix entre les deux pronoms, ceux où l'anglais utilise le pronom we. On exclura donc de l'analyse les emplois de nous comme pronom disjoint (avec nous), dans les composés nous-mêmes et nous autres et dans les structures clivées (c'est nous qui...), de même que les emplois non restreints de on, où le sujet est inclus dans un groupe spécifique, connu ou non (ici on parle français), ou dans l'humanité tout entière (on aura tout vu !). Ces emplois de l'un ou l'autre pronom étant obligatoires dans leurs contextes respectifs, ils seront enseignés en temps utile, comme tout autre aspect de la grammaire française.

De même, on laissera de côté la structure l'on, surtout utilisée à l'écrit en registre soigné, bien qu'elle soit également présente, d'après Coveney (2004), en écrit familier (recherche Google) et en français oral (7, $3 \%$ de tous les $(l)$ 'on produits par des journalistes à la télévision, utilisant ou non un téléprompteur, et 1, $2 \%$ dans un corpus de Picardie). Utilisée surtout après $s i$ et que relatif pour des raisons euphoniques (éviter l'hiatus ou l'allitération, parfois gênante, qu'on con...), un peu comme la liaison ou le /t/ de va-t-on, cette structure est par ailleurs d'ordre phonétique, qui n'est pas pertinent ici.

\section{Historique}

Pour comprendre comment on a fini par concurrencer nous pour exprimer $1 \mathrm{PL}$ à l'oral, il convient de faire un retour en arrière sur l'origine du phénomène, à partir de comédies ou remarques de grammairiens, qui nous renseignent utilement sur les états de langue antérieurs à l'époque moderne. C'est ce qu'ont fait King et al. (2011) dans une analyse très détaillée, dont nous reprenons une partie ici.

Notons tout d'abord que nous (< latin nos) a toujours existé dans l'histoire du français : "Et nos somes ci an poverte " Nous sommes ici en pauvreté' (Chrétien de Troyes, Chevalier au lion, Folio 99, vers 5309, XIIe s.). Il a d'abord été concurrencé par $j(e)$ (< latin ego) suivi d'une forme verbale en -ons : "Moi et le gros Lucas, je nous amusions à bâtifoler avec des mottes de tarre. " (Molière, Don Juan, Acte II, Sc. 1, 1665). Limité aux classes populaires et jugé comme tel par les grammairiens du XVI et du XVII siècles, je 1PL était encore majoritaire au XVII ${ }^{e}$ et au XVIII ${ }^{e}$, avant de devenir 
carrément marginal au $\mathrm{XIX}^{\mathrm{e}}$ puis inexistant au $\mathrm{XX}^{\mathrm{e}}$, sauf dans certaines régions du Poitou et de l'Acadie, où l'on peut encore l'entendre aujourd'hui. Il résulte de cette quasi-disparition que la seule opposition intéressante pour $1 P L$ aujourd'hui est entre nous et on.

L'origine de on est nominale (cas sujet du latin homo, devenu hum, um, puis on en ancien français, alors que le cas régime hominem a donné homme), ce qu'on note également dans d'autres langues comme le danois, l'islandais et l'allemand (man), ou le portugais brésilien (a gente). Sa valeur non restreinte, qui persiste aujourd'hui (on frappe à la porte), a d'abord été la seule possible : "Si cum om per dreit son fradra salvar dift " 'Comme on doit secourir son frère' (Serments de Strasbourg 842). Puis, le pronom a pris diverses valeurs restreintes, en remplacement d'autres pronoms, pour exprimer « la modestie, la discrétion, l'ironie, le mépris, l'orgueil, le reproche, etc. " (Grevisse 1969 : paragr. 587) : "Il est grand besoing qu'on [= je] le dye» (Farce de Maistre Pathelin 1486) ; "A-t-on [= tu] été sage, mon enfant ?" (Musset, Rhin allemand, 1841) ; "Commandez qu'on [= elle, Junie] vous aime et vous serez aimé » (Racine, Britannicus, II, 2, 1669) ; "Pour Dieu, mes amys, qu'on [= vous] me dye " (Farce du pourpoint rétrée, $\left.\mathrm{XV}^{\mathrm{e}}\right)$; "Ceste femme par son plaisir/A la ville le gardera/La ou on [= nous] le visitera/Moy et vous, chascune sepmaine " (Gautier de Coinci, Les miracles de Notre-Dame, $\left.\mathrm{I}, 364, \mathrm{XIII}^{\mathrm{e}}\right)$. On était même à la mode dans la bonne société du XVII ${ }^{\mathrm{e}}$ siècle, jusqu'à ce que cette dernière se rende compte qu'il avait une connotation populaire, bonne raison pour l'éviter.

L'emploi restreint de on est resté rare jusqu'au XIX ${ }^{\mathrm{e}}$ siècle, puis il est devenu de plus en plus fréquent dans le vernaculaire, au point de remplacer nous dans certains milieux défavorisés. Il s'est étendu aux classes favorisées plus tard, sans toutefois éliminer tout à fait nous, qui avait déjà éclipsé je au siècle précédent. On peut dire que la diffusion sociale de on restreint est complète depuis le milieu du $\mathrm{XX}^{\mathrm{e}}$ siècle et qu'il est devenu la forme 1PL normale de la conversation courante, reléguant nous aux contextes plus formels du registre soutenu.

Cette conclusion est étayée par les commentaires de nombreux grammairiens du $\mathrm{XX}^{\mathrm{e}}$ siècle. Frei (1929) considère que on 1PL appartient pleinement au système pronominal du parler populaire. Pour Brunot (1933 : 379), " aujourd'hui, on, pourchassé par les professeurs de purisme, est extrêmement usité dans les milieux populaires. C'est un mot commode qui tend à se répandre partout au lieu de la première personne ". Et ceci pas uniquement dans les milieux populaires : "On s'est presque substitué à nous dans l'usage parlé, même dans le discours familier des personnes cultivées " (von Wartburg et Zumthor 1958 : paragr. 688). Même Grevisse, qui décrit on 1PL comme une vulgarité de langage jusqu'en 1975 (10 édition du Bon usage), finit par concéder que on se substitue largement à nous, et que le phénomène a même atteint la langue littéraire, surtout quand elle veut paraître familière. 
On retiendra de ce bref survol historique que l'emploi de on 1PL n'est pas récent, puisqu'il remonte au moins au XII ${ }^{e}$ siècle, qu'il n'est pas limité au parler populaire, ni à la langue orale, et qu'il est devenu majoritaire depuis déjà longtemps en français oral.

Ce glissement dans l'expression de 1PL ne s'est pas fait par hasard. D'abord, on existait déjà dans le système. Le remplacement d'un pronom par un autre est commun, comme on peut le voir dans les exemples ci-dessus ou dans la langue anglaise actuelle (Each student will have to submit their [= his/her] application). Il permet aussi au locuteur de se désengager pour éviter des choix délicats (Bonjour Suzanne, on $[=\mathrm{tu} / \mathrm{vous}]$ arrive en avance aujourd'hui ?) ou de rester dans le vague tout en laissant entendre le précis (On [= ils/nous] n'aime pas ça en haut lieu). Par ailleurs, il existe une grande similarité sémantique entre le sens non restreint de on et celui de nous ( $A u$ Québec, on produit/nous produisons du sirop d'érable), qui favorise le passage de l'un à l'autre. Sur le plan morphologique, l'économie réalisée par l'usage de on 1PL n'est pas négligeable : cinq formes verbales au lieu de six ; remplacement d'une terminaison peu fréquente en -ons par la forme du singulier, mieux connue et surtout plus simple, puisqu'il s'agit en fait d'une absence de terminaison (je, tu, il, elle, on /рав1/). De même, la forme d'insistance nous, on évite la cacophonie nous, nous et s'inscrit aisément dans le paradigme du singulier moi, je; toi, tu; lui, il. Enfin et surtout, l'ascension rapide de on 1PL au XIX ${ }^{\mathrm{e}}$ siècle correspond à une période de grands bouleversements sociaux (mobilité sociale et migration urbaine interne favorisant le nivellement), qui ont accéléré la désintégration du système morphosyntaxique synthétique du français vers la structure analytique de la fin du XIX ${ }^{\mathrm{e}}$ siècle : pronom sujet obligatoire et perte des terminaisons verbales, devenues redondantes. Au présent de l'indicatif, il ne reste plus guère que 2PL à l'oral (vous VERBE-ez) pour empêcher que cette désintégration soit complète.

On aura compris, d'après ce qui précède, que, bien que la forme nous soit parfaitement adéquate pour exprimer $1 \mathrm{PL}$, la concurrence de on est tout à fait naturelle.

\section{Situation actuelle}

Les études consacrées au pronom 1PL et mentionnées dans le tableau 1 ci-dessous confirment le remplacement presque total de nous par on, le premier étant réservé aux situations formelles (Coveney 2000) et covariant avec d'autres marqueurs de formalité (ne... pas, vous) ou certains facteurs qui la favorisent (âge avancé, classe favorisée, débuts d'interview). Ce remplacement, dans la mesure où les différences entre catégories sont représentatives du français général, varie en fonction inverse de l'âge des locuteurs et de leur penchant pour la formalité, ces deux traits étant rassemblés dans la catégorie "politiciens » de Söll (1974) et chez les 
enseignants de Fonseca-Greber (2003) et de Coveney (2000), où nous reste fréquent.

\begin{tabular}{|c|c|c|c|c|}
\hline Contexte & Auteur & Corpus & Catégorie & on 1PL \\
\hline FL1 France & $\begin{array}{l}\text { Söll } 1974 \\
\text { Coveney } 2000 \\
\text { Fonseca-Greber/ } \\
\text { Waugh 2003 } \\
\text { King et al. } 2011 \\
\\
\\
\text { Compernolle (sous } \\
\text { presse) }\end{array}$ & $\begin{array}{l}\text { Picardie } \\
\text { France/Suisse } \\
\text { Orléans } 1969 \\
\text { (+ textes littéraires } \\
\text { depuis le XVII' + } \\
\text { grammairiens } \\
\text { France ('chat') }\end{array}$ & $\begin{array}{l}\text { Enfants } \\
\text { Classe moyenne } \\
\text { Politiciens } \\
\text { Jeunes }\end{array}$ & $\begin{array}{l}100 \% \\
80 \% \\
60 \% \\
95,6 \% \\
99 \% \\
55-100 \% \\
96,1 \%\end{array}$ \\
\hline FL1 Canada & \begin{tabular}{|l|} 
Laberge 1977 \\
Deshaies 1991 \\
Nadasdi 2005 \\
Rehner et al. 2003 \\
\end{tabular} & $\begin{array}{l}\text { Montréal } \\
\text { Québec } \\
\text { Québec } \\
\text { Ontario }\end{array}$ & $\begin{array}{l}\text { Jeunes } \\
\text { Jeunes }\end{array}$ & \begin{tabular}{|l|}
$98,4 \%$ \\
$99,5 \%$ \\
$98 \%$ \\
$99 \%$ \\
\end{tabular} \\
\hline
\end{tabular}

Tableau 1: On 1PL en français langue première (FL1)

Pour vérifier l'affirmation de Claire Blanche-Benveniste, selon laquelle " tous les locuteurs emploient actuellement on pour nous, y compris les hommes politiques dans leurs discours publics de quelque tendance qu'ils soient" (Blanche-Benveniste 1997 : 40), nous avons examiné un corpus de parole présidentielle destiné au départ à une analyse phonétique (Thomas, à paraître). Chez le président de Gaulle, pendant un entretien télévisé d'une heure en période de faible popularité électorale (1965), le nous reste nettement majoritaire (75 occurrences), à côté de 4 cas de l'on non restreint qui renforcent la formalité. Mais on 1PL est largement représenté (34 occurrences), notamment dans la séquence suivante : "De 1920 à 1940, on a eu 47 ministères en 20 ans. Voilà le régime des partis. Alors, naturellement, on a été battus, écrasés. En 40, on n'avait rien préparé, on était divisés par les partis. On n'avait pas les armes nécessaires ». Les deux formes semblent alterner librement, en particulier dans comme nous le faisions autrefois et comme on le faisait autrefois, tous deux présents dans le même entretien. Et ceci chez un locuteur qui, avec ses passés simples, ses imparfaits du subjonctif et ses liaisons facultatives rares, ne donne pas facilement dans la familiarité. Un demi-siècle plus tard, chez le président Sarkozy, enregistré en contexte identique en 2013, c'est au contraire on qui l'emporte (81 occurrences, contre 58 nous et un seul l'on). Mais on trouve, là encore, des passages où les deux formes alternent librement, notamment dans les exemples suivants : " [...] nous avons rencontré les partenaires sociaux, et toutes ces mesures, on les a 
discutées avec eux. [...] On a décidé de prendre le taureau par les cornes et, là aussi, dès le mois de février, nous voterons un texte... " Sans s'attarder sur la valeur de données qui ne concernent que deux individus, et ne sont donc pas forcément représentatives d'un style ou d'une époque, on ne peut s'empêcher de constater que on 1PL est d'un usage tout à fait normal, même aux niveaux les plus élevés de la hiérarchie française.

Quant à la dimension canadienne du phénomène, importante puisque nos étudiants auront probablement plus de contacts avec les Québécois qu'avec les Français (voyages, échanges, affaires), les données du tableau 1 montrent clairement que on 1PL écrase encore plus nous qu'en France. Cela s'explique probablement par le mélange de dialectes et de classes sociales caractérisant les colons de la Nouvelle-France et offrant les conditions parfaites pour un nivellement vers la forme dominante de l'époque (on), devenue écrasante dès le XIX ${ }^{e}$ d'après Blondeau (2003). On peut noter par ailleurs que le français canadien a toujours été moins sujet aux pressions élitistes, favorables à nous, que le français de France. Cette remarque vaut d'ailleurs également pour un autre français " périphérique ", celui de la Suisse, où l'usage de on 1PL est aussi plus élevé qu'en France, d'après Fonseca-Greber et Waugh (2003). Enfin, pour ce qui est de la forme je + VERBE-ons, qui survit encore en Acadie, notamment à Pubnico et Chéticamp en NouvelleÉcosse, elle remonte, d'après l'Atlas linguistique de la France, aux colons du Centre-Ouest qui ont fondé l'Acadie. C'est cette forme, en tout cas, et pas nous, qui a pu faire concurrence à on en Acadie, jusqu'à récemment.

\section{Nous/on en pédagogie du FL2}

Afin de déterminer jusqu'à quel point les manuels de FL2 représentent la réalité décrite ci-dessus, nous avons examiné le traitement de 1PL dans quinze d'entre eux, publiés ces dix dernières années et envoyés à notre département d'Études françaises à des fins promotionnelles. Tous visent l'enseignement du français aux niveaux débutant ou intermédiaire, à l'écrit et à l'oral, ou du moins ils n'excluent pas ce dernier aspect explicitement. On pourrait donc s'attendre à ce que la prédominance de on 1PL soit adéquatement illustrée dans ce corpus, qui est assez représentatif des publications existantes dans le domaine. Le résultat de cet examen permet de classer les manuels en quatre catégories :

On mentionné avec $i l$, elle, sans détails :

Oates et Dubois. 2003. Personnages (Houghton Mifflin)

Furry et Jarausch. 2007. Bonne continuation (Pearson Prentice Hall)

Thomason et Hirsch. 2003. Ensuite (McGraw Hill)

Blood et Mobarek. 2004. Intrigue (Pearson Prentice Hall) 


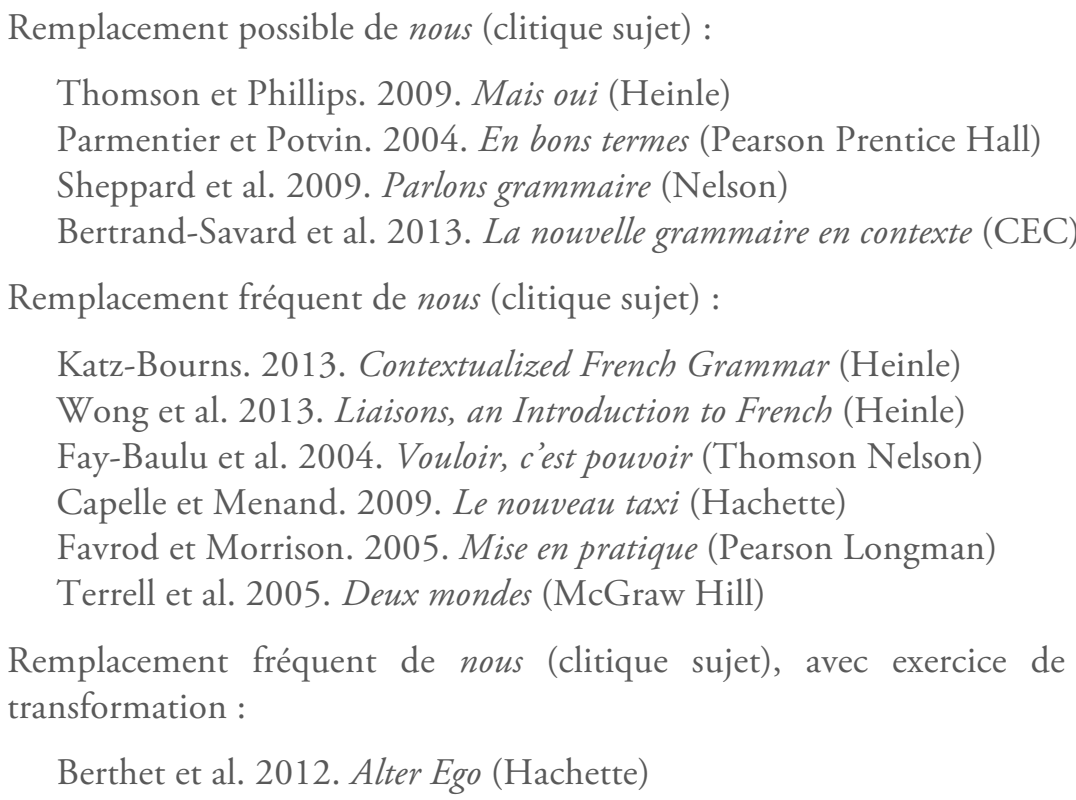

On voit d'après cette classification que la moitié des manuels, les huit premiers, ne représentent pas adéquatement le remplacement de nous par on, qui est beaucoup plus que "possible ", et qu'un quart d'entre eux ne le mentionnent pas du tout. Dans l'autre moitié, où l'on admet que ce remplacement est "fréquent ", un seul texte passe à la pratique, c'est-à-dire considère on 1PL comme une acquisition valable. Dans l'ensemble, nous sommes donc bien loin de la réalité actuelle de 1PL, telle qu'elle a été décrite ci-dessus, bien que la chronologie de ces manuels suggère une lueur d'espoir, les ouvrages les plus progressistes étant aussi, en général, les plus récents.

Le problème n'est pas limité au niveau universitaire, où on pourrait arguer que la langue écrite est naturellement privilégiée en prévision des cours de littérature, de linguistique et de traduction offerts à partir de la deuxième année. Une analyse approfondie des manuels utilisés dans les programmes d'immersion à l'élémentaire (Rehner et al. 2003, voir le tableau 2) montre que on $1 \mathrm{PL}$ y reste minoritaire, même dans les dialogues. Et si la proportion de on $1 \mathrm{PL}$ atteint $56 \%$ dans la performance orale des élèves, c'est plus par imitation du modèle omniprésent fourni par leurs enseignants, souvent francophones ( $83 \%$ de on $1 P L)$, que par l'étude du français dans les manuels disponibles. L'importance du contact avec les francophones est confirmée par une autre observation des auteurs de cette étude : l'emploi de on 1PL augmente après un échange linguistique au Québec ou en France. Ceci pourrait expliquer par ailleurs la grande variation individuelle notée par les auteurs, car le séjour linguistique en milieu francophone n'est pas universel en milieu immersif. 


\begin{tabular}{|c|c|c|c|c|}
\hline Contexte & Auteur & Corpus & Catégorie & on $1 \mathrm{PL}$ \\
\hline FL2 immersion & Rehner et al. 2003 & $\begin{array}{l}\text { Ontario } \\
\text { (anglophones) }\end{array}$ & $\begin{array}{l}\text { Élèves } \\
\text { Enseignants } \\
\text { Manuels } \\
\quad \text { (dialogues) } \\
\quad \text { (textes) }\end{array}$ & $\begin{array}{l}56 \% \\
83 \% \\
48 \% \\
17 \%\end{array}$ \\
\hline FL2 université & $\begin{array}{l}\text { Sax } 2001 \\
\text { Dewaele } 2002\end{array}$ & $\begin{array}{l}\text { USA } \\
\text { (anglophones) }\end{array}$ & $\begin{array}{l}2^{\mathrm{e}} \text { année, aucun échange } \\
4^{\mathrm{e}} \text { année, } 50 \% \text { échange } \\
\text { Master, } 100 \% \text { échange } \\
\text { Oral } \\
\text { Écrit }\end{array}$ & $\begin{array}{l}60 \% \\
4 \% \\
57 \% \\
81 \% \\
79,6 \% \\
78,1 \%\end{array}$ \\
\hline
\end{tabular}

Tableau 2 : On 1PL en français langue seconde (FL2)

Au niveau universitaire, l'analyse du parler des étudiantes américaines enregistrées par Sax (2001) va dans le même sens. Le gouffre entre les étudiantes qui n'ont pas fait d'échange ( $4 \%$ de on 1 PL en $2^{\mathrm{e}}$ année) et celles qui en ont fait un (81\% au niveau master) en dit long sur l'importance du contact avec les francophones, même si ce n'est pas le seul facteur qui ait pu contribuer à un si grand écart. L'étude révèle même une corrélation entre la durée du séjour en France et l'emploi de on 1PL. Par ailleurs, l'auteure a noté une différence entre l'imitation d'un dialogue avec une colocataire française et l'imitation d'une entrevue formelle (moins d'occurrences de on 1PL), ce qui montre que les étudiantes étaient conscientes de la variation linguistique caractérisant 1 PL.

L'étude de Dewaele (2002), portant sur des étudiants néerlandophones de niveau intermédiaire FL2 ou FL3, relève aussi une corrélation entre l'emploi de on 1PL et l'utilisation du français oral, que ce soit par contact direct avec des francophones ou par exposition à la télévision en français, mais aucune avec le sexe ou la classe sociale des sujets, ni avec la durée de leur scolarité en français. Ce dernier point suggère que la scolarité ne fait que renforcer l'emploi de nous et que des contacts avec les francophones sont nécessaires pour augmenter l'emploi de on $1 \mathrm{PL}$, comme il a déjà été signalé ci-dessus. La quasi-égalité des données à l'écrit et à l'oral surprend quelque peu, dans la mesure où l'on s'attendrait à ce que la conscience d'une certaine variation, démontrée par Sax, s'applique à la différence de formalité entre les deux codes. À moins que cette conscience ne s'éveille qu'à partir d'un certain niveau, qui dépasserait celui des étudiants " intermédiaires " observés par Dewaele.

On voit donc, d'après les observations pédagogiques présentées ici, que les étudiants de FL2 sont plutôt mal préparés en classe à l'usage réel des pronoms 1PL. Ce constat est illustré par la remarque anecdotique suivante, 
provenant d'une participante à un forum électronique sur la question : «None of my French teachers mentioned anything about the rare usage of nous in modern conversational French» (Flennie, sur www.slowtalk.com/groupee/forums/a/tpc/f/27710511321/m/520003627001).

La situation n'est pas très différente quand on sort de la classe pour consulter les grammaires en ligne. Les quelques sites visités à ce sujet, à partir d'une simple recherche Google, se répartissent aussi entre ceux qui ne mentionnent pas du tout l'option on $1 \mathrm{PL}$ et ceux qui reconnaissent sa fréquence.

Que faire dans la salle de classe, devant tant de divergences chez les spécialistes?

\section{5. Ébauche de solutions pédagogiques}

Le premier pas vers la réhabilitation de on 1PL en classe est sans doute l'acceptation du français ordinaire oral comme sujet légitime d'enseignement du FL2, avec ses variations sociales, géographiques et stylistiques. L'époque où l'on enseignait d'abord les langues secondes comme accès privilégié à la littérature et à la culture est révolue. Même si cet aspect est encore important dans nos universités, nos étudiants préfèrent en général un enseignement plus pratique, qui leur sera utile pendant leurs voyages ou séjours à l'étranger, de plus en plus faciles et fréquents aujourd'hui, ou dans leurs contacts avec les médias francophones.

S'il s'agit donc de privilégier la communication avec le monde francophone contemporain, par rapport à l'appréciation de sa culture, il faut d'abord s'informer comment les francophones communiquent aujourd'hui, à l'écrit et surtout à l'oral. D'où le recours aux travaux des linguistes, abondamment cités ici, dont le rôle est justement de documenter l'état actuel de la langue, sans jugement ni interdiction, contrairement aux grammairiens prescriptivistes, qui ne s'intéressent qu'au «bon » usage, c'est-à-dire celui de l'élite, inspiré de la langue écrite et modèle privilégié par les manuels scolaires.

L'analyse linguistique révèle que la langue est généralement sujette à variation, comme le souligne Labov (1972), contrairement à l'impression de fixité donnée par l'enseignement traditionnel du FL2. Dans le cas qui nous intéresse ici, l'alternance nous/on n'est qu'un exemple d'une sorte de diglossie " prestige/ordinaire " également présente dans ne...pas/pas, cela/ça, quatrelquat', tableltab', ils sont_[t]_arrivés/i sont arrivés ou (seulement au Canada) je vais/je vas. Dans tous les cas, la variante de prestige est nettement minoritaire dans l'usage. Peut-on décemment continuer à l'enseigner en FL2 à l'exclusion de la variante dominante ? Ceci d'autant plus que les étudiants qui nous arrivent $\mathrm{du}$ secondaire, du moins en Ontario, sont censés être équipés des compétences sociolinguistiques évoquées ici (Ministère de l'Éducation 2000). 
Ce n'est pas que les apprenants soient incapables de maitriser la variation, car la recherche en acquisition des langues a démontré (voir détails dans Rehner et al. 2003) que certains facteurs influençant le choix des variantes en L1 sont également pertinents pour la L2. Mais ces facteurs mènent rarement à un usage substantiel du vernaculaire en L2, où les variantes de prestige dominent, et ceci à un niveau nettement supérieur à celui de la L1. Ce n'est généralement qu'à la faveur de contacts hors classe avec des francophones, notamment au cours de séjours linguistiques en France ou au Québec, que les étudiants acquièrent véritablement les variantes non standard du français.

La première solution pratique au problème de l'enseignement du français ordinaire serait donc d'encourager les séjours linguistiques en milieu francophone ou, au moins, d'organiser des contacts suivis avec les francophones disponibles sur place, notamment les assistants à qui nous confions traditionnellement nos séminaires, laboratoires ou activités socioculturelles en français.

En l'absence de contacts substantiels avec des francophones, il faudrait au moins que les enseignants soient éclairés par suffisamment de connaissances linguistiques pour tenir compte de la performance et des fréquences d'utilisation de certaines variantes, plutôt que du prestige de la langue classique. De tels enseignants préféreraient sans doute travailler avec des documents en rapport plus étroit avec la langue contemporaine, comme ceux développés par Valdman (1998), Cuq et Gruca (2005) ou Lyster (2007), qui ont intégré des styles de parole informelle dans leurs matériaux.

Si on est peu enclin à explorer ces documents, on devrait au moins choisir un manuel de classe qui est en harmonie avec les réalités linguistiques contemporaines (voir la section 4 ci-dessus). C'est d'ailleurs dans un tel manuel qu'on a le plus de chances de trouver des exercices utiles. On découvre ainsi, dans Alter Ego, A1, déjà mentionné ci-dessus, l'exercice de transformation suivant pour l'alternance nous/on:

Transformez avec le pronom on.

- Qu'est-ce que vous faites le week-end?

- Quand nous sommes libres, nous sortons avec des amis. Nous avons rendez-vous en centre-ville. Nous allons dans un bar sympa, puis nous dînons dans un restau, et nous passons le reste de la soirée dans une boîte. Nous rentrons à la maison vers 6 heures du matin, fatigués mais contents! (61)

À noter que l'exercice est assorti d'éléments familiers ou neutres cohérents avec on, tant sur le plan de la syntaxe (Qu'est-ce que vous faites? au lieu de Que faites-vous ?) que du vocabulaire (sympa, restau, boîte), et typiques 
du parler de la jeunesse française d'aujourd'hui avec laquelle nos étudiants espèrent communiquer.

Et si le manuel de classe choisi ou imposé n'offre aucun exercice pertinent, il est facile d'en créer en utilisant on à la place du nous habituel, comme dans l'exercice structural élémentaire suivant :

$$
\begin{aligned}
& \text { - Jennifer, tu as fini? } \\
& \text { - Oui, j’ai fini. } \\
& \text { - Et toi, Susan? } \\
& \text { - J'ai fini aussi. } \\
& \text { - Alors toutes les deux...? } \\
& \text { - On a fini. }
\end{aligned}
$$

On remarquera que, quel que soit le contexte d'apprentissage et les documents utilisés, les solutions suggérées ci-dessus ont toutes en commun le respect de l'usage réel du français par les francophones.

\section{Conclusion}

Le survol historique et surtout l'état présent de l'emploi des pronoms on $1 \mathrm{PL}$ présentés ci-dessus démontrent clairement que le remplacement de nous par on est presque total en français oral contemporain, reléguant nous aux domaines formels du discours et à la langue écrite. Cette réalité, qui n'est ni anecdotique, ni récente, ni artificielle, devrait a priori être reconnue partout dans la francophonie, s'il est vrai qu'une langue se définit par l'usage qu'en font ses locuteurs.

Or, il y a parfois loin de la réalité à la perception, car

\footnotetext{
la soumission du français à la variation ne correspond pas forcément à l'image que ses locuteurs ou que ses apprenants s'en font. En France, en particulier, c'est la variété écrite, et particulièrement littéraire, de la langue qui a forgé chez ses usagers les représentations dominantes de la langue, ou norme (Cuq et Gruca 2005 : 81).
}

L'oral est souvent l'objet de perceptions négatives, à cause de ses connotations populaires. Dans le cas précis de 1PL, il peut aussi y avoir une certaine hésitation à utiliser ce on parfois ambigu (sens restreint ou non ?), alors que les choses étaient claires tant qu'on se limitait à l'opposition on (non restreint)/nous (restreint). Ainsi, l'enseignement traditionnel de la grammaire est basé sur la langue écrite, surtout littéraire, et sur une terminologie grammaticale vieille de deux millénaires, et il est caractérisé par le 
développement systématique des compétences langagières dans le contexte prescriptif et moral du "bon " usage (l'écrit), parfaitement illustré dans l'ouvrage idoine de Grevisse. On comprend alors que l'auteure du dernier site mentionné ci-dessus commence sa leçon avec l'avertissement : «The French you learn is not always the French we speak in France ».

Cette approche prescriptive est légitime dans les milieux francophones, où la langue orale est déjà largement acquise quand les enfants commencent l'école et où on a surtout besoin des ouvrages de grammaire pour améliorer la compétence écrite. Elle l'est beaucoup moins en contexte FL2, où tout est à apprendre, à commencer par la communication de base, orale ou écrite. Et cette communication inclut différents niveaux de formalité: présentations, entrevues, réunions, conversations téléphoniques, contacts familiers, entre autres, qui ne sont pas souvent pris en compte par les matériels méthodologiques.

Il convient donc de réorienter notre enseignement en fonction des réalités du français ordinaire décrites par les linguistes, aussi bien pour nous/on que pour les autres variables évoquées ici. Cela ne signifie pas la condamnation des formes de prestige, mais seulement leur relégation à une place secondaire, cohérente avec le statut marginal qu'elles occupent en français contemporain. C'est uniquement à ce prix que l'on pourra faciliter le contact de nos étudiants, surtout les plus avancés, avec l'univers francophone qu'ils ont choisi d'explorer.

\section{Références bibliographiques}

Blanche-Benveniste, C. 1997. Approches de la langue parlée en français. Paris: Ophrys.

Blondeau, H. 2003. "The Old nous and the New nous. A Comparison of $19^{\text {th }}$ and $20^{\text {th }}$ Century Spoken Quebec French”. University of Pennsylvania Working Papers in Linguistics 9 (2): $1-15$.

Boutet, J. 1986. «La référence à la personne en français parlé : le cas de on ». Langage et société $38: 19-50$.

Brunot, F. 1933. Précis de grammaire historique de la langue française. Paris: Masson et Cie.

van Compernolle, R. A. 2008. "Nous vs. on: Pronouns with First-Person Plural Reference in Synchronous French Chat". Canadian Journal of Applied Linguistics 11(2) : 85-100.

Coveney, A. 2000. "Vestiges of nous and the $1^{\text {st }}$ Person Plural Verb in Informal Spoken French". Language Sciences 22 : 447-81.

Coveney, A. 2004. "The Alternation between l'on and on in Spoken French". Journal of French Language Studies 14 (2) : 91-112.

Critchley, S. 1994. "Discourse Variety in Contemporary French Language: A Pedagogical Approach”. Dans Discourse Variety in Contemporary French: Descriptive and Pedagogical Approaches, sous la direction de J.A. Coleman et R. Crawshaw. London : Association for French Language Studies and Centre for Information on Language Teaching : 203-236.

Cuq, J.-P. et I. Gruca. 2005. Cours de didactique du français langue seconde. Grenoble : Presses universitaires de Grenoble. 
Deshaies, D. 1991. "Contribution à l'analyse du français québécois : étude des pronoms personnels ». Revue québécoise de linguistique théorique et appliquée 10 : 11-40.

Dewaele, J.-M. 2002. "Using Sociolinguistic Variants in Advanced French Interlanguage: The Case of nous versus on". EUROSLA Yearbook. Amsterdam : John Benjamins : 205-226.

Frei, H. 1929. La grammaire des fautes. Genève : Slatkine Reprints.

Fonseca-Greber, B. et L.R. Waugh. 2003. "On the Radical Difference between the Subject Personal Pronouns in Written and Spoken European French”. Corpus Analysis: Language Structure and Language Use. Amsterdam : Rodopi : 225-254.

Grevisse, M. 1969. Le bon usage, neuvième édition. Gembloux : Duculot.

King, R., Martineau, F. et R. Mougeon. 2011. "The Interplay of Internal and External Factors in Grammatical Change : First-Person Plural Pronouns in French". Language 87 (3) : 470-509.

Labov, W. 1972. Sociolinguistic Patterns. Philadelphie: University of Pennsylvania Press.

Laberge, S. 1977. Etude de la variation des pronoms définis et indéfinis dans le français parlé à Montréal. Thèse de doctorat, Université de Montréal.

Lyster, R. 1999. "Diffusing Dichotomies: Using the Multidimensional Curriculum Model for Developing Analytic Teaching Materials in Immersion”. Dans French Second Language Education in Canada: Empirical Studies, sous la direction de S. Lapkin. Toronto et Buffalo: University of Toronto Press : 197-218.

Lyster, R. 2007. Learning and Teaching Languages through Content: A Counterbalanced Approach. Amsterdam : John Benjamins.

Nadasdi, T. 2005. "Le français en Ontario ". Dans Le français en Amérique du Nord : État présent, sous la direction de A. Valdman, J. Auger et D. Piston-Hatlen. Québec : Presses de l'Université Laval : 99-115.

Ontario Ministry of Education. 2000. The Ontario Curriculum Grades 11 and 12: French as a Second Language - Core, Extended, and Immersion French. Toronto : Queen's Printer.

Rehner, K, Mougeon R. et T. Nadasdi. 2003. "The Learning of Socio-Stylistic Variation by Advanced FSL Learners: The Case of nous versus on in Immersion French". Studies in Second Language Acquisition $25: 127-156$.

Sax, K. 2001. "Stylistically Speaking: Variable Use of nous versus on in American Learners' French". Communication présentée au 30 ${ }^{\text {th }}$ NWAVE Colloquium, Raleigh, 11-14 octobre.

Söll, L. 1974. Gesprochenes und geshribenes Französish [Français oral et écrit]. Berlin : Erich Schmidt Verlag.

Thomas, A. À paraître. "Aspects phonologiques de la parole présidentielle ". Dans Actes du $X X X I V^{e}$ colloque international d'Albi : langages et significations; discours d'autorité et discours de l'autorité, sous la direction de P. Marillaud.

Valdman, A. 1998. "La notion de norme pédagogique dans l'enseignement du français langue étrangère ". Dans Analyse linguistique et approches de l'oral : recueil d'études offert en hommage à Claire Blanche-Benveniste, sous la direction de M. Bilger, K. Vanden Eynde et F. Gadet. Paris et Louvain : Peeters : 177-178.

von Wartburg, W. et P. Zumthor. 1958. Précis de syntaxe du français contemporain. Berne : Francke. 